\title{
Towards an Automatic Data Value Analysis Method for Relational Databases
}

\author{
Malika Bendechache ${ }^{\mathrm{a}}{ }^{\mathrm{a}}$, Nihar Sudhanshu Limaye and Rob Brennan $\oplus^{\mathrm{b}}$ \\ ADAPT Centre, School of Computing, Dublin City University, Dublin 9, Ireland
}

Keywords: $\quad$ Data Value, RDB, Information Systems, CMM, Metrics, Survey.

\begin{abstract}
Data is becoming one of the world's most valuable resources and it is suggested that those who own the data will own the future. However, despite data being an important asset, data owners struggle to assess its value. Some recent pioneer works have led to an increased awareness of the necessity for measuring data value. They have also put forward some simple but engaging survey-based methods to help with the first-level data assessment in an organisation. However, these methods are manual and they depend on the costly input of domain experts. In this paper, we propose to extend the manual survey-based approaches with additional metrics and dimensions derived from the evolving literature on data value dimensions and tailored specifically for our use case study. We also developed an automatic, metric-based data value assessment approach that (i) automatically quantifies the business value of data in Relational Databases (RDB), and (ii) provides a scoring method that facilitates the ranking and extraction of the most valuable RDB tables. We evaluate our proposed approach on a real-world RDB database from a small online retailer (MyVolts) and show in our experimental study that the data value assessments made by our automated system match those expressed by the domain expert approach.
\end{abstract}

\section{1 - INTRODUCTION}

With recent advances in information and communication technologies, such as digital sensor technologies, social media and digital transformation of organisations, we are able to collect huge amounts of data across a wide variety of fields. Furthermore, we live in an era where all what we do is leaving a digital footprint (data) which can be recorded, collected and used to provide insights (Bendechache et al., 2019).

Storing huge amounts of data and selecting which part is worth curating or using to drive the analytics process can be very costly for companies (Brous et al., 2016). This requires a form of data governance to decide which data and business management actions should be conducted (Khatri and Brown, 2010). The data management association, DAMA, defines data governance "as the processes, policies, organisation and technologies required to manage and ensure the availability, usability, integrity, consistency, auditability and security of data" (International, 2017). The basis of governance decision making is balanc-

a (iD https://orcid.org/0000-0003-0069-1860

b (D) https://orcid.org/0000-0001-8236-362X ing value and risk (Tallon, 2013). This means it is important to have methods to decide which data is valuable and which data is not (International, 2017). Data value can play a deciding role in data management decisions as it can be used to rank the data based on its importance to the organisation, thus facilitating the decision-making process for data deletion, curation and improvement (Attard and Brennan, 2018).

Unfortunately research into data value is immature, despite notable contributions from practitioners (Laney, 2017). To date, most work has focused on establishing the dimensions of data value (Viscusi and Batini, 2014) rather than developing concrete metrics or assessment methods as is seen in the related domain of data quality research (Zaveri et al., 2016). One fruitful area of work has been on manual data value assessment techniques (Brennan et al., 2019) but these are not scalable to the typical data governance challenges being faced by organisations today and will not satisfy the realtime, automated data value monitoring and control loops for the next generation of data governance systems (Brennan et al., 2018) based on the systems of insight paradigm for digital transformation. However many potential data value metrics overlap with data value 
metrics (Laney, 2017) and there have been a number of siloed data management applications developed independent of any overall governance system that include data value calculations in their core, for example in file management (Wijnhoven et al., 2014) applications. Thus, for practical business applications that are still overwhelmingly driven by data stored in relational databases there is a lack of clarity on how data value or data governance may be deployed to improve decision making.

The aim of this work is to answer the two following research questions: (i) To what extent can the data value of a relational database $(R D B)$ of an organisation be accurately quantified (measured) using a metrics-based approach?, and (ii) Which existing data value metrics are most effective at predicting the value of data in a Relational database (RDB)?

To answer these two questions, we first propose an extended questionnaire (survey) for manual data value assessment of an RDB. The questions were designed based on a set of relevant data value dimensions for any RDB-including the real world use case of an online retailer studied in this paper. The extended survey targets data value dimensions that are now considered important but were omitted from the original one. The survey was answered by domain experts in an organisation and scored following the method of Sadjko et al. to manually create a baseline data value assessment for the organisation. Second, we propose a new automatic metrics-based approach to assessing the business value of an RDB. Our approach was evaluated on real RDB data provided by MyVolts, a lean online retail SME which has largely automated its business and stores all its business process data in its RDB. Our automatic data value assessment technique consists of a system that queries the RDB to calculate metrics for each data value dimension assessed by the answers to the relevant questions in the survey provided to the domain expert. Our goal is to show that our automated technique can match the data value assessment baselines provided by the domain experts. We also propose a scoring method for data assessment results which assigns a score to the different tables in the RDB and returns a ranking of the most valuable table amongst them. Additionally, we integrate our proposed approach with a wellknown visualisation tool to provide faster and simpler insights into the data value assessment results.

This paper makes the following contributions:

1. Proposing an extended questionnaire for manual data value assessment.

2. Proposing an automatic and quantified metricbased approach for data value assessment in an RDB.
3. Comparing the results of both manual and automatic data value assessment techniques.

4. Proposing a scoring method for data asset value.

5. Integrating a visualisation tool (Tableau) to show the final scoring system.

The remainder of this paper is organised as follows: Section 2 introduces value-driven data governance and data value dimensions. Section 3 presents selected related work. Section 4 explains our extended survey-based approach, whereas Section 5 details our proposed automatic data value analysis approach. Section 6 describes our use case based on the RDB of the company MyVolts. Section 7 presents and discusses the experimental results. The paper concludes with a summary of the paper and a discussion of future work in Section 8.

\section{BACKGROUND}

\subsection{Value-driven Data Governance}

As the volume of data continues to rise, and enterprises/organisations rely more and more on their data, this latter is being duplicated, transformed, fragmented, and isolated into silos (El Abed, 2009). The sheer volume of data also affects data quality, since data standards cannot be enforced so easily. Such data may lead to additional risks, increased costs, inefficient processes, and therefore potential business losses (Brous et al., 2016). Although there is a general understanding that high quality data leads to more benefits when the data is exploited, there is a lack of maturity of data management processes, as well as the resulting business impact of using it. These issues already show a clear need to manage data assets. Since data governance, management efforts, and investments are on the rise, it is becoming increasingly relevant to identify the economic value of data and its return on investment (Laney, 2017). Note that the value of the data can also be determined by how data is used -for example for control and coercion (Barn and Barn, 2018). In this paper we focus on the the monetary value of data. Data value has been used as a basis for organisational decision making for quality management (Even and Shankaranarayanan, 2005), but also as a part of automated control systems for data lifecycles (Chen, 2005). Failing to assign value to data will result in a number of consequences such as retaining information that has little to no value, reducing data usage, and leaving data investments vulnerable to budget cuts (Maina, 2003). Hence, the data value is an aspect that plays a very important role in 
data governance. The issue is that although data is gaining recognition as a valuable asset, it has as yet resisted reliable quantitative measurement.

\subsection{Data Value Dimensions}

Dimensions are the set of measurable features that characterise data value, similar to data quality dimensions (International, 2017). A dimension is a category typically designed while considering useroriented views towards data value. Data value assessment should aim to be holistic in measuring the dimensions of data value for an organisation (Brennan et al., 2019). For example, one of the dimensions which are mentioned in the literature is "Volume" (Laney, 2017). The volume dimension reifies concepts such as "more data more value" and leads to metrics such as counting joins in queries and an indicator of value whereby if these two things are satisfied by a database table then its value is high compared to other tables (Attard and Brennan, 2018).

\section{LITERATURE REVIEW}

Trends such as Big Data have popularised the need for enterprises to become more data-driven and increased the need for a better understanding of what that means (Economist, 2017). However, deciding which data is valuable is a difficult task. The information has a notional value only. People think it is valuable but they can not quantify its value (Moody and Walsh, 1999).

(Moody and Walsh, 1999) identify "Laws of information" to understand data value. However, the authors proposed a manual survey approach towards data valuation and did not provide any concrete metrics to quantify the data value for a specific data asset.

The book "Infonomics: How to Monetize, Manage, and Measure Information as an Asset for Competitive Advantage" by (Laney, 2017), discusses the different quantitative data value approaches. However, the book looked at the metrics from a business point of view. Besides, the survey is shallow and unsatisfying in terms of pointing out operational approaches to quantitative data value methods and tools when compared with equivalent work on data quality metrics.

The main method to assess data value is through finding dimensions of information value (Brennan et al., 2018). A couple of works have been done with this regards in different application domains. For example, (Sajko et al., 2006) provides an idea of dimensions and their corresponding metrics to be used for value assessment. The main dimension discussed by (Sajko et al., 2006) are Rebuilding, Legislative, Time, and Market value. However, these metrics can vary according to the use case at hand. (Wijnhoven et al., 2014) used mainly the dimension Volume for assessing the data value in the area of file-storage management. Assessing the data value in the information management lifecycle was done by (Chen, 2005). The author looked specifically at Usage as a data value dimension. (Rao and $\mathrm{Ng}, 2016$ ) looked at the value assessment in information pricing. Data governance (Stander, 2015), and data quality management (Even et al., 2010) are two other areas that looked at value assessment. A recent paper by (Brennan et al., 2018) includes a Capability Maturity Model (CMM) that tries to identify specific metrics and uses open data to analyse it.

In this paper we use the metrics defined by the CMM model defined by (Brennan et al., 2018) and test them on a relational database environment. We propose an automated metric-based system that quantifies the business value of an RDB and compare its results to the baseline survey-based approach.

\section{AN EXTENDED SURVEY-BASED APPROACH}

Manual data value assessment techniques based on surveys are an important tool in the absence of automated techniques. In fact, in the book Infonomics (Laney, 2017) several aspects of data value are identified as best quantified through surveys, for example data usability, given their complex relation to business processes. Here we present an extended survey technique that addresses more dimensions of data value.

One structured data value survey method that has already been deployed on many industrial use cases (Brennan et al., 2019) is the security risk assessment survey of Sadjko et al. (Sajko et al., 2006). This simple five question survey is structured around the five data value dimensions of utility, replacement cost, competitive advantage, regulatory risk and timeliness. It is important to note that each question and set of five possible answers is phrased in ways that are approachable to business domain experts rather than data governance personnel. The specific data value dimensions are never named. For example for the regulatory risk dimension: "Is there any obligation to keep this data and any consequences for the organisation if it loses it?" with possible answers "(a) There are none. (b) It is necessary to keep the data for a brief period. (c) The organisations should keep the data but 
without consequences. (d) Keeping the data is obligatory and the company can suffer sanctions. (e) Keeping the data is obligatory and the sanctions are strict." A Likert-type scoring scale is also provided for the questionnaire results by Sajko et al. to convert the survey results into numerical scores for each dataset. The structured questionnaire is used to drive a stakeholder assessment of the importance (value) of organisational data assets as part of a workshop to determine which assets should receive the most attention in the creation of a data security solution.

However there are limitations to this survey method. The set of dimensions selected seems arbitrary given recent research on data value dimensions (Viscusi and Batini, 2014). For example it is a major limitation to not assess the usage dimension, which is identified in both old (Moody and Walsh, 1999) and new works (International, 2017) as a key dimension of data value. In addition, the contribution of data quality to value is not assessed. This is an important omission because data quality metrics are mature and available for reuse, and crop up frequently in surveys of the dimensions of data value (Viscusi and Batini, 2014). One of the key dimensions of data value identified for Big Data, but more widely applicable, is the volume of data (one of the three V's of Big Data) (Laney, 2017). Finally we add the dimension of security as data which is protected by security measures in an organisation is more likely to have been assessed as valuable or important. For each of these dimensions a new, business user-friendly, survey question was developed (see Table 1). All of these dimensions of data value were identified as relevant to our case study.

Having a wider variety of data value dimensions addressed by the survey allows for more discriminatory power but it also enables us to tailor the questionnaire to a specific use case. All of the questions developed here are widely applicable to practical data value assessments for many types of dataset and domain. Although manual data value assessment survey methods rely heavily on human expertise, their simplicity and engaging nature is very attractive for deploying a first-level data assessment method in an organisation to (i) establish baselines for the evaluation of automated methods, (ii) act as a first assessment of data value from local domain experts that are aware of the business use of data assets but who may struggle with linking value either to more abstract data value dimensions or choosing appropriate data value metrics, and (iii) to stimulate organisational awareness of data value. Although (Sajko et al., 2006) report that the method has been applied many times, their work provides no example data on responses.
Figure 1 provides an overview of our extended manual data value assessment survey method. In order to save the answers for the set of questions, a questionnaire form was created using Google form. The Google form link is shared with domain experts in the organisation (company). These questions were mapped to a set of dimensions and metrics in the data. Replies to the Google form are saved as a CSV file to be used later as a baseline for comparing results of our proposed automatic metric-based data value approach.

\begin{tabular}{|c|} 
Dimension/Metric \\
Design
\end{tabular}$\rightarrow \begin{gathered}\text { Questionnaire } \\
\text { Creation } \\
\text { (Google Form) }\end{gathered} \rightarrow \begin{gathered}\text { Questionnaire } \\
\text { Result } \\
\text { Extraction (CSV } \\
\text { File) }\end{gathered} \rightarrow \rightarrow \begin{aligned} & \text { Relational } \\
& \text { Database } \\
& \text { Storage }\end{aligned}$

Figure 1: An Overview of the Survey-based Approach.

\section{AN AUTOMATIC DATA VALUE METRIC-BASED ANALYSIS APPROACH}

The goal of the proposed automatic metric-based algorithm is to propose an automatic way of assessing and quantifying the business value on any RDB. A query-based technique is proposed to find the business value of each table in an RDB. These values will be compared to the values returned by the baseline (survey-based) technique. Afterwards, a scoring system is proposed to assign a value score for each table in the RDB. Finally, a visualisation dashboard is proposed to display the final results.

The first step in developing the automated method was to establish a set of metrics that could be used to generate indicators for each data value dimension to be assessed in the RDB (see Table 2). Then a set of competency questions were developed for each metric to identify the RDB system attributes that would need to be exposed by queries of the database, logs or metadata. Once these were established, a further level of specific query development can be carried out to develop queries for the specific RDB SQL queries (see Table 3).

The proposed automated evaluation process has a set of steps starting from data gathering and cleaning until data value scoring visualisation:

1. Data Cleaning. The data used is cleaned using an open-source data cleaning tool called OpenRefine to remove any inconsistency (unknown characters, distorted values).

2. Data Storing. An RDB is created to store the datasets that we want to assess its business value. The CSV file that contains the survey questions 
Table 1: Extended Survey-based Questions.

\begin{tabular}{|l|l|l|}
\hline \multicolumn{1}{|c|}{ Dimension } & New & \multicolumn{1}{c|}{ Question } \\
\hline Utility & No & What happens if you do not have this data anymore? \\
\hline Replacement Cost & No & What is the cost of replacing this data or producing new equivalent data? \\
\hline Legislative Risk & No & Is there any obligation to keep this data and any consequences for the organisation if it loses it? \\
\hline Timeliness & No & Does the data value fall in the course of time? \\
\hline Competitive Advantage & No & What happens if your competitor has the same data? \\
\hline Quality & Yes & What happens if this data is incomplete, inconsistent, inaccurate or invalid? \\
\hline Usage & Yes & How frequently is this data accessed? \\
\hline Volume & Yes & How big is this data? \\
\hline Security & Yes & What security measures are deployed for this data? \\
\hline
\end{tabular}

Table 2: Proposed Relational Database Metrics for Each Data Value Dimension.

\begin{tabular}{|l|l|l|}
\hline \multicolumn{1}{|c|}{ Dimension } & \multicolumn{1}{c|}{ Metric } & \multicolumn{1}{c|}{ Competency Question } \\
\hline Utility & Number of business processes & Which table has the most different systems querying it? \\
\hline Replacement Cost & Reproduction & Which table has a capacity of new data to produce new data? \\
\hline Legislative Risk & Confidential Data & Which data loss will create legal consequences if organisation losses it? \\
\hline Timeliness & Shelf Life Reduces & Which database table information value falls in course of time? \\
\hline Competitive Advantage & Unique Data & what database is unique for the organisation? \\
\hline \multirow{2}{*}{ Quality } & Duplicate Records & Which table has the least duplicates? \\
\cline { 2 - 3 } & Null Records & Which database table has least null values? \\
\hline Usage & Number of writes in a day & Which database table is used more frequently in a day? \\
\hline \multirow{2}{*}{ Volume } & High Record Count & Which database table stores the highest records? \\
\cline { 2 - 3 } & Interconnection & Which database table can join with most of the other data? \\
\hline Security & Encryption & Which database table contains encrypted values? \\
\hline
\end{tabular}

and answers created in the survey-based approach is also loaded into the database.

\section{Data Value and Scoring.}

-A Query System: once the data is stored, the algorithm starts querying the RDB. It fires SQL queries specifically designed in line with survey questions to get results. The query results will be compared to the survey results for accuracy (matching). E.g. Survey Question: Which database stores the highest records? Survey Answers - Answer Table 1. SQL query: Query COUNT to get the highest record count of database table - Answer Table 1. Similarly, other queries are designed for each metric/question. The results will be compared to the result of a survey-based approach.

-A Scoring System: the algorithm uses a scoring system, where it assigns a single point for each successful output. In other words, If both Survey answers and query result matches then the algorithm adds a point to the table that scores best on each question. (See Table1). E.g. The survey answer, as well as query answer, says that Table 1 has the highest record count. Hence, Table 1 will score 1 point. If a table satisfies more metrics, more points are added against it. Finally, the table with the highest points is returned to be the most valued one.

4. Data Visualisation. The algorithm connects the database tables and the data value scoring system to a visualisation dashboard to display the ranking of each database table along with its value. The visualisation tool used is Tableau and it displays the results in the form of graphs that simplify results' understanding.

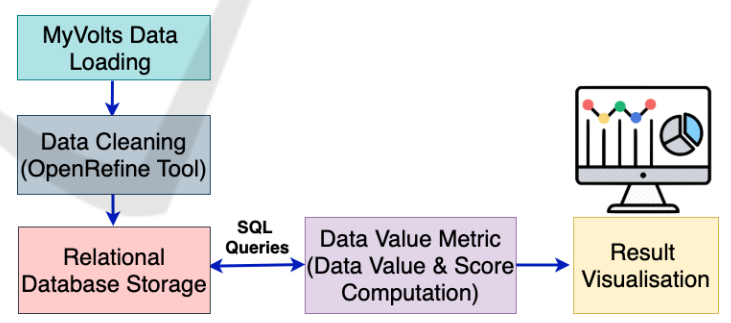

Figure 2: An Overview of the Automatic Metric-based Approach.

\section{USE CASE: MyVolts}

To show the results of our automatic metric-based assessment technique, we consider a real use case of an RDB used by MyVolts.com (MyVolts). The MyVolts $^{1}$ is an online retail a Small Medium Enterprise (SME) based in Dublin. MyVolts focuses on providing modern and innovative power solutions for home, mobile and professional devices - mainly for musi-

\footnotetext{
${ }^{1}$ http://myvolts.com/article.php/8/about_myvolts
} 
cians and other mobile creatives. The company focuses on both customer engagement and product expertise. They aim to provide well-designed and flexible power delivery solutions to exactly meet the needs of many customers who pass through their checkouts annually. the company has served over 1 million customers to date.

MyVolts produces and processes a huge amount of data. The company requires multiple databases to store their data. MyVolts has two main data resources; 1) the main database (main DB) hosted locally by MyVolts which contains several tables, and 2) an Amazon seller database (Amazon DB) which is hosted in the cloud.

The RDBs in MyVolts contains multiple tables. In this paper, we only consider the relevant tables to our case study which are; Price and Sales along with Amazon DB. Each table in MyVolts database contains thousands of records.

- Amazon Database. It contains a table named amazonuk_listings which stores the electric items sold by MyVolts.com on the Amazon website. The table includes; the item price, the number of items, the last updated price for an item, etc. We will refer to this table with the name Amazan DB.

- Pricing Database. Contains a table that stores the pricing of items available at MyVolts. The company has a strategy of lowering the item's price if it is not sold for a long duration. So it contains price data along with the discount percentage to get a new price. We will refer to this table with the name Price DB.

- Sales Database. This database contains a table that stores the personal details of customers that buy the MyVolts products online. We will refer to this table with the name Sale DB.

Storing a huge amount of records (data) can be problematic for MyVols. Therefore, this work offers the company a new and automatic approach to assessing the business value of their data stored in their RDBs. This will help the company understand their data and help them manage their resources.

\section{EXPERIMENTAL RESULTS}

In this section, we will show the experimental results of both extended survey-based and automatic metricbased approaches for data value assessment. The proposed approaches were tested on data provided by MyVolts. The goals are (1) to compare the surveybased and the automatic metric-based approaches and show that the automatic approach succeeds to match the results of the manual survey-based approach, (2) to show that the proposed automatic metric-based approach not only returns good results but it also returns a scoring system that enables us to rank the database tables and return the most valuable amongst them to the organisation, in our case MyVolts company.

\subsection{Survey-based vs Metric-based Data Value Results}

The algorithm starts by querying the MyVolts database tables according to dimensions and metrics already defined in section 1 . The algorithm fires some SQL queries specifically designed in line with survey questions to get results. E.g. Query COUNT to get the highest record count of database table. Likewise, multiple queries are designed according to metrics and dimensions. Table 3 summarises the set of the used SQL queries to answer to the questions defined in Table 1.

The results of the comparison between the manual survey-based approach and the automatic metricbased approach are shown in Table 4.

As it can be seen from Table 4, the automatic metric-based approach results match those of the manual survey-based approach across most of the dimension in an RDB data source. The automatic metric-based approach succeeded to assess the data value of dimensions like Rebuilding, Security, Volume, Usage, Quality, and Legislative. However, metrics of dimensions like Time and Market value could not be measured by the proposed automatic approach. This is because the dimension "Market value" needs contextual information and the dimension "Time" could not be measured without extra data (i.e. metadata). Therefore, more information or metadata besides the RDB need to be taken into account to cover all the defined metric dimensions.

Figure 3 shows the value of each of the MyVolts database tables. As we can see, the Price DB clearly has more value points comparing to the other two databases. Hence we can conclude that the Price DB is more valuable for the MyVolts company than the two other database tables.

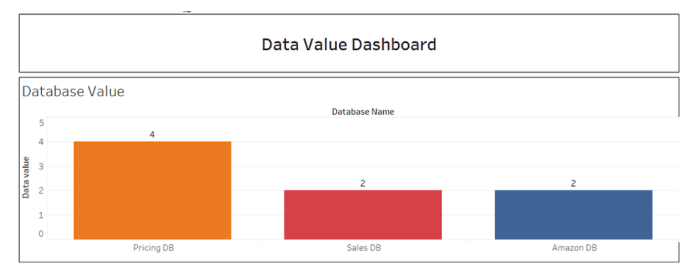

Figure 3: The Automatic Data Value Assessment Results. 
Table 3: Description of used SQL queries.

\begin{tabular}{|c|c|c|c|c|c|c|}
\hline \multicolumn{2}{|c|}{ Dimension } & Possible Questions Generated & \multicolumn{4}{|c|}{ Description of Used SQL Queries } \\
\hline \multicolumn{2}{|c|}{ Replacement Cost } & $\begin{array}{l}\text { Which table has a capacity to pro- } \\
\text { duce new data? }\end{array}$ & \multicolumn{4}{|c|}{$\begin{array}{l}\text { Table with the largest average new } \\
\text { data per day }\end{array}$} \\
\hline \multicolumn{2}{|l|}{ Security } & $\begin{array}{l}\text { Which database table contains en- } \\
\text { crypted values? }\end{array}$ & \multicolumn{4}{|c|}{$\begin{array}{l}\text { Table with the largest number of en- } \\
\text { crypted values }\end{array}$} \\
\hline \multirow{2}{*}{\multicolumn{2}{|c|}{ Volume }} & $\begin{array}{l}\text { which database table stores the } \\
\text { highest records? }\end{array}$ & \multirow{2}{*}{\multicolumn{4}{|c|}{$\begin{array}{l}\text { Table with the largest number of } \\
\text { values } \\
\text { Table with the largest number of } \\
\text { join }\end{array}$}} \\
\hline & & $\begin{array}{l}\text { Which database table can join with } \\
\text { most of the other data? }\end{array}$ & & & & \\
\hline \multicolumn{2}{|l|}{ Usage } & $\begin{array}{l}\text { Which database table is used more } \\
\text { frequently in a day? }\end{array}$ & \multicolumn{4}{|c|}{$\begin{array}{l}\text { Table with the largest number of } \\
\text { access queries per day (could be } \\
\text { stored in a table or in a server log) }\end{array}$} \\
\hline \multirow{2}{*}{\multicolumn{2}{|c|}{ Quality }} & $\begin{array}{l}\text { Which table has the least dupli- } \\
\text { cates? }\end{array}$ & \multicolumn{4}{|c|}{$\begin{array}{l}\text { Table with the lowest number of du- } \\
\text { plicate values }\end{array}$} \\
\hline & & $\begin{array}{l}\text { Which database table has the least } \\
\text { null values? }\end{array}$ & \multicolumn{4}{|c|}{$\begin{array}{l}\text { Table with the lowest number of } \\
\text { null values }\end{array}$} \\
\hline \multicolumn{2}{|c|}{ Legislative Risk } & $\begin{array}{l}\text { Which data loss will create legal } \\
\text { consequences if organisation losses } \\
\text { it? }\end{array}$ & \multicolumn{4}{|c|}{$\begin{array}{l}\text { Table containing the largest num- } \\
\text { ber of attributes from specified } \\
\text { list (Name, Bank Details, Address, } \\
\text { etc.) }\end{array}$} \\
\hline \multicolumn{2}{|l|}{ Time } & $\begin{array}{l}\text { Which database table information } \\
\text { value falls in course of times? }\end{array}$ & \multicolumn{4}{|c|}{ NA } \\
\hline \multicolumn{2}{|c|}{ Competitive Advantage } & $\begin{array}{l}\text { What database table is unique for } \\
\text { the organisation? }\end{array}$ & \multicolumn{4}{|l|}{ NA } \\
\hline \multicolumn{7}{|l|}{ 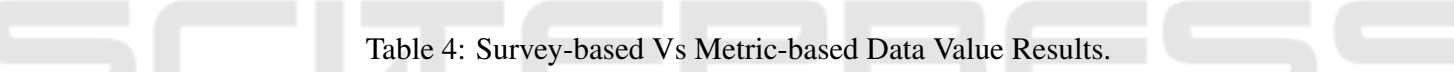 } \\
\hline Dimension & & Possible Questions Generated & & $\begin{array}{l}\text { Survey-based } \\
\text { Answers }\end{array}$ & $\begin{array}{l}\text { Metric-based } \\
\text { Answers }\end{array}$ & Match \\
\hline Replacement Cost & Which ta & has a capacity to produce new data? & & Pricing DB & Pricing DB & Yes \\
\hline Security & Which d & base table contains encrypted values? & & Sales DB & Sales DB & Yes \\
\hline \multirow{2}{*}{ Volume } & \multicolumn{3}{|c|}{ which database table stores the highest records? } & Amazon DB & Amazon DB & Yes \\
\hline & \multicolumn{3}{|c|}{ Which database table can join with most of the other data? } & Pricing DB & Pricing DB & Yes \\
\hline Usage & \multicolumn{3}{|c|}{ Which database table is used more frequently in a day? } & Amazon DB & Amazon DB & Yes \\
\hline \multirow{2}{*}{ Quality } & \multirow{2}{*}{\multicolumn{2}{|c|}{$\begin{array}{l}\text { Which table has the least duplicates? } \\
\text { Which database table has the least null values? }\end{array}$}} & & DB & Pricing DB & Yes \\
\hline & & & & $\mathrm{DB}$ & Pricing DB & Yes \\
\hline Legislative & \multicolumn{3}{|c|}{ Which data loss will create legal consequences if organisation losses it? } & Sales DB & Sales DB & Yes \\
\hline Timeliness & \multicolumn{3}{|c|}{ Which database table information value falls in course of times? } & Amazon DV & NA & $\mathrm{NA}$ \\
\hline Competitive Advantage & \multicolumn{3}{|c|}{ What database table is unique for the organisation? } & Pricing DB & NA & NA \\
\hline
\end{tabular}

\section{CONCLUSION AND FUTURE WORK}

State-of-the-art works on data value assessment analysis are very limited. The few existing works are focused on manual survey-based approaches. Although the survey-based approaches proved their efficiency, they lack in terms of quantifying the business value of data for an organisation. In this paper, we proposed an automatic metric-based approach that can quantify the business value of a given RDB source for an organisation. An extended version of a manual survey-based approach was also proposed and used as a baseline to compare the results of our automatic based approach. The results satisfy the first research question as "To what extent can the data value of an RDB data source of an organisation be accurately measured using a metrics-based approach?". Our experimental results showed that nearly $70 \%$ of metrics used to measure data value give positive results. Metrics of dimensions like Rebuilding, Security, Volume, Usage, Quality, and Legislative are successful for measuring data value. However metrics of dimensions like Time, Market value failed to measure data value for an RDB data source because Market value and Time need more specifics and contextual information. Furthermore, the results also answered the second research question "Which predefined data value metrics are most effective at predicting a value for the MyVolts online retailer use case?". Eight dimen- 
sion were used in this paper among which five (Rebuilding, Usage, Legislative, Market value, and Time) are the predefined ones taken from the state-of-the-art metrics and three are the extra tailored dimension Volume, Security, and Quality, designed specifically for MyVolts use case. The algorithm returned a perfect match compared to the manual survey-based approach results and it succeeded to accurately measure the value of an RDB data source for a given use case of MyVolts with predefined dimensions/metrics like Rebuilding, Legislative and Usage and with tailored dimensions like Security, Volume and Quality.

As future work, we plan to validate the proposed approach with other real world use cases. We also plan to study the type of metadata that need to be joined to the RDB data and means to combined them to enable measuring of the Market-value and Time dimensions. Furthermore, we also plan to propose another multi-criteria decision analysis approach for assessing more accurately the business value of data for an organisation.

\section{ACKNOWLEDGEMENTS}

The ADAPT Centre is funded under the SFI Research Centres Programme (Grant 13/RC/2106) and is cofunded under the European Regional Development Fund.

\section{REFERENCES}

Attard, J. and Brennan, R. (2018). Challenges in valuedriven data governance. In OTM Confederated International Conferences" On the Move to Meaningful Internet Systems", pages 546-554. Springer.

Barn, B. and Barn, R. (2018). Towards a unified conceptual model for surveillance theories:" we shall meet in the place where there is no darkness"-1984, george orwell. In 2018 IEEE/ACM 40th International Conference on Software Engineering, pages 71-80. IEEE.

Bendechache, M., Tari, A.-K., and Kechadi, M.-T. (2019). Parallel and distributed clustering framework for big spatial data mining. International Journal of Parallel, Emergent and Distributed Systems, 34(6):671-689.

Brennan, R., Attard, J., and Helfert, M. (2018). Management of data value chains, a value monitoring capability maturity model. In ICEIS, pages 573-584.

Brennan, R., Attard, J., Petkov, P., Nagle, T., and Helfert, M. (2019). Exploring data value assessment: a survey method and investigation of the perceived relative importance of data value dimensions. In ICEIS 201921 st International Conference on Enterprise Information Systems, pages 200-207. SciTePress.

Brous, P., Janssen, M., and Vilminko-Heikkinen, R. (2016). Coordinating decision-making in data management activities: a systematic review of data governance principles. In International Conference on Electronic Government, pages 115-125. Springer.

Chen, Y. (2005). Information valuation for information lifecycle management. In Second International Conference on Autonomic Computing (ICAC'05), pages 135-146. IEEE.

Economist, T. (2017). The world's most valuable resource is no longer oil, but data. The Economist: New York, $N Y, U S A$

El Abed, W. (2009). Data governance: a business valuedriven approach. In white paper.

Even, A. and Shankaranarayanan, G. (2005). Value-driven data quality assessment. In $I C I Q$.

Even, A., Shankaranarayanan, G., and Berger, P. D. (2010). Evaluating a model for cost-effective data quality management in a real-world crm setting. Decision Support Systems, 50(1):152-163.

International, D. (2017). DAMA-DMBOK: Data Management Body of Knowledge. Technics Publications.

Khatri, V. and Brown, C. V. (2010). Designing data governance. Communications of the ACM, 53(1):148-152.

Laney, D. B. (2017). Infonomics: How to Monetize, Manage, and Measure information as an Asset for competitive advantage. Taylor \& Francis.

Maina, C. (2003). Valuing information in an information age: The price model and the emerging information divide among individuals, societies, and nations. In Proceedings of the Annual Conference of CAIS/Actes du congrès annuel de l'ACSI.

Moody, D. L. and Walsh, P. (1999). Measuring the value of information-an asset valuation approach. In ECIS, pages 496-512.

Rao, D. and Ng, W. K. (2016). Information pricing: a utility based pricing mechanism. In 2016 IEEE 14th Intl Conf on Dependable, Autonomic and Secure Computing-(DASC), pages 754-760. IEEE.

Sajko, M., Rabuzin, K., and Bača, M. (2006). How to calculate information value for effective security risk assessment. Journal of Information and Organizational Sciences, 30(2):263-278.

Stander, J. B. (2015). The modern asset: big data and information valuation. $\mathrm{PhD}$ thesis, Stellenbosch: Stellenbosch University.

Tallon, P. P. (2013). Corporate governance of big data: Perspectives on value, risk, and cost. Computer, 46(6):32-38.

Viscusi, G. and Batini, C. (2014). Digital information asset evaluation: Characteristics and dimensions. In Caporarello, L., Di Martino, B., and Martinez, M., editors, Smart Organizations and Smart Artifacts, pages 7786. Springer International Publishing.

Wijnhoven, F., Amrit, C., and Dietz, P. (2014). Value-based file retention: File attributes as file value and information waste indicators. Journal of Data and Information Quality (JDIQ), 4(4):1-17.

Zaveri, A., Rula, A., Maurino, A., Pietrobon, R., Lehmann, J., and Auer, S. (2016). Quality assessment for linked data: A survey. Semantic Web, 7(1):63-93. 